\title{
Liver injury in COVID-19 patients with metabolic syndrome-a narrative review
}

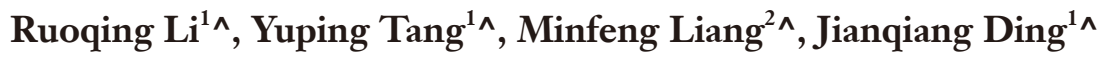 \\ ${ }^{1}$ Department of General Medicine, Chongqing University Central Hospital, Chongqing Emergency Medical Center, Chongqing, China; \\ ${ }^{2}$ Department of Infectious Diseases, The First People's Hospital of Foshan, Foshan, China \\ Contributions: (I) Conception and design: All authors; (II) Administrative support: M Liang, J Ding; (III) Provision of study materials or patients: R Li, \\ Y Tang; (IV) Collection and assembly of data: R Li, Y Tang; (V) Data analysis and interpretation: R Li, Y Tang, M Liang; (VI) Manuscript writing: \\ All authors; (VII) Final approval of manuscript: All authors. \\ Correspondence to: Jianqiang Ding. Department of General Medicine, Chongqing University Central Hospital, Chongqing Emergency Medical \\ Center, \#1 Health Road, Yuzhong District, Chongqing 400014, China. Email: jding18@foxmail.com.
}

\begin{abstract}
Objective: To comprehensively analyze that how liver injury in patients with metabolic syndrome is affected by coronavirus disease 2019 (COVID-19) and provide clinical reference to their prevention and treatment.

Background: The current COVID-19 pandemic poses a major threat to human life and health. Metabolic syndrome is also a major global health problem, and evidence suggests that patients with metabolic syndrome are at an increased risk of COVID-19 complications. Liver injury is one of the main manifestations of extrapulmonary organ injury in patients with COVID-19. Currently, the effects of metabolic syndrome on liver injury in patients with COVID-19 are unclear.

Methods: In this study, we searched the PubMed, Embase, and China National Knowledge Infrastructure (CNKI) databases for articles on the latest developments of liver injury in COVID-19 patients with metabolic syndrome from 2019 to comprehensively analyze the current knowledge of how liver injury in patients with metabolic syndrome is affected by COVID-19. We used the free key words "metabolic syndrome" OR "hypertension” OR “obesity" OR “diabetes” OR “dyslipidemia” OR "liver injury" OR "SARS-CoV-2" OR "COVID-19" in all fields. We imposed no language restrictions.

Conclusions: Both COVID-19 and metabolic syndrome and its components are closely related to liver injury, may induce liver injury through direct or indirect mechanisms. Therefore, when COVID-19 is combined with metabolic syndrome, it may increase the risk of liver injury, and it cannot be ruled out that the two diseases have a superimposed effect on liver injury.
\end{abstract}

Keywords: Metabolic syndrome; coronavirus disease 2019 (COVID-19); liver injury

Submitted May 10, 2021. Accepted for publication Jun 24, 2021.

doi: 10.21037/apm-21-1398

View this article at: https://dx.doi.org/10.21037/apm-21-1398

\section{Introduction}

Coronavirus disease 2019 (COVID-19) has presented major challenges to the fields of public health prevention and control all over the world and remains a serious threat to human health. COVID-19 has become a global pandemic and the current coronavirus outbreak is due to the severe acute respiratory syndrome coronavirus 2 (SARS-CoV-2) that was defined by the International Committee on Taxonomy of Viruses on February 11th, 2020 (1-4). The clinical symptoms of SARS-CoV-2 infection have been

^ ORCID: Ruoqing Li, 0000-0001-6706-7339; Yuping Tang, 0000-0002-8417-7836; Minfeng Liang, 0000-0002-9199-7867; Jianqiang Ding,

0000-0003-1964-1513. 
defined by the World Health Organization (WHO) as COVID-19, which also declared the COVID-19 pandemic in March 2020 (5). Daily epidemiological monitoring statistics from the WHO show that as of January 7th 2021, there were more than 85 million confirmed cases with more than 1.87 million deaths resulting from COVID-19 worldwide. A large number of studies have shown that metabolic syndrome increases the susceptibility of patients to COVID-19 (6-9).

Metabolic syndrome is a combination of multiple risk factors characterized by hypertension, diabetes, dyslipidemia, and obesity. Saklayen et al. estimated that approximately $25 \%$ of the global population suffers from metabolic syndrome (10). Studies have shown that metabolic syndrome affects around $25 \%$ of the world's population (11) with $12-37 \%$ of the Asian population being affected and $12-26 \%$ of the European population being affected (12). The high incidence of metabolic syndrome creates severe challenges in the prevention and treatment of chronic diseases in the field of public health, and these challenges are significantly enhanced by the current COVID-19 pandemic. Patients with metabolic syndrome often also suffer from fatty liver disease and have abnormally high levels of transaminase. Additionally, COVID-19 can cause different degrees of liver injury resulting in acute liver failure, which is more common in critically ill COVID-19 patients than ordinary COVID-19 patients (13-15). Metabolic syndrome and COVID-19 are closely related to liver injury; however, there were several reviews on the relationship between COVID-19 and liver injury, but their attention is paid to the impact of COVID-19 on liver injury and its possible mechanisms. There is a lack of in-depth analysis of this common and complicated situation when COVID-19 is combined with metabolic syndrome, especially lack of relevance analysis between the various metabolic components and liver injury. In this study, we searched the PubMed, Embase, and China National Knowledge Infrastructure (CNKI) databases for articles on the latest developments of liver injury in COVID-19 patients with metabolic syndrome from 2019 to comprehensively analyze the current knowledge of how liver injury in patients with metabolic syndrome is affected by COVID-19. We present the following article in accordance with the Narrative Review reporting checklist (available at https://dx.doi. org/10.21037/apm-21-1398).

\section{COVID-19, metabolic syndrome, and liver injury}

Angiotensin-2 converting enzyme (ACE2) receptor is a functional receptor on the cell surface. It has been confirmed that SARS-COV-2 virus can invade cells in the body through the ACE2 receptor and cause the occurrence of COVID-19 (16). At the same time, ACE2 is an important regulator of the renin-angiotensin-aldosterone system (RAAS). After SARS-COV-2 virus enters the body, it breaks the balance of RAAS system-related hormones and activates RASS, leading to the further progress of COVID-19 (16). The main clinical symptoms of COVID-19 and the injury of various organs and tissues may also be related to the distribution and expression of ACE2 receptors in the body.

Respiratory system injury is the most common clinical manifestation of COVID-19. However, SARS-CoV-2 infection can cause systemic damages, including myocardial and liver injury, vasculitis, multiple system organ failure, and even death in severe cases (17-19). At present, there are many reports on the liver injury related to COVID-19, but the description of hepatitis has not yet been defined. The main manifestations of liver dysfunction include increased levels of aspartate aminotransferase, alanine transaminase (ALT), gamma-glutamyl transferase, alkaline phosphatase, and total bilirubin (TBIL) (20). Liver injury is diagnosed when any of the above liver function indices is more than twice the upper limit of the normal range. A clinical study of Chinese COVID-19 sufferers showed that $15.4 \%$ of patients also suffered from liver injury (21).

At present, the mechanism by which COVID-19 causes liver injury is not very clear; however, several potential mechanisms have been proposed. The first mechanism involves ACE2 receptors that are expressed at significantly higher levels in the cells of the bile ducts $(60 \%)$ than the hepatocytes (3\%) (22-24). SARS-CoV-2 binds to the ACE2 receptor of cells in the bile ducts by binding to spike viral proteins; thus, the virus could directly enter the target cells and cause liver injury (25). Second, as COVID-19 injury to the respiratory system is severe, this could lead to hypoxia in hepatocytes that consequently increases the expression of ACE2 receptors and hypoxia-inducible factors (HIFs) $(24,26)$. As HIFs are closely related to hepatocyte metabolism, this could result in hepatic steatosis and liver injury (26). Third, SARS-CoV-2 infection activates autoimmunity and inflammation in vivo that causes significant increases in the expression of many cytokines, such as interleukin-6 (IL-6) and interleukin-2 (IL-2) (27-29). These elevated cytokine levels lead to an imbalance of the innate immune response in the liver (hepatic innate immunity) and the activation of natural immune cell mass (30). These changes may result in necrosis of the liver tissue, hepatocyte proliferation, and the upregulation of 
ACE2 causing autoimmune injury to the liver (31). Fourth, the drugs recommended for the treatment of COVID-19, such as lopinavir/ritonavir and chloroquine, could lead to liver injury. Finally, SARS-CoV-2 infection could also enhance previous chronic liver disease.

Metabolic syndrome consists of a combination of risk factors that result in cardiovascular disease, such as hyperglycemia, elevated blood pressure, dyslipidemia, (hypertriglyceridemia and low high-density lipoprotein cholesterol), and obesity (32). The metabolic syndrome can be diagnosed by any three or more of the following (33): (I) blood glucose greater than $5.6 \mathrm{mmol} / \mathrm{L}(100 \mathrm{mg} / \mathrm{dL})$ or drug treatment for elevated blood glucose; (II) HDL cholesterol $<1.0 \mathrm{mmol} / \mathrm{L}(40 \mathrm{mg} / \mathrm{dL})$ in men, $<1.3 \mathrm{mmol} / \mathrm{L}$ $(50 \mathrm{mg} / \mathrm{dL})$ in women or drug treatment for low HDL-C; (III) blood triglycerides $>1.7 \mathrm{mmol} / \mathrm{L}(150 \mathrm{mg} / \mathrm{dL})$ or drug treatment for elevated triglycerides; (IV) waist $>102 \mathrm{~cm}$ (men) or $>88 \mathrm{~cm}$ (women); (V) blood pressure $>130 / 85 \mathrm{mmHg}$ or drug treatment for hypertension. Metabolic syndrome is considered an important risk factor in the occurrence and development of COVID-19 (34). A study conducted in the United States (US) involving 1,482 COVID-19 patients showed that around $12 \%$ of hospitalized patients had concomitant diseases. In this group of COVID-19 patients, $49.7 \%$ had hypertension, $28.3 \%$ had diabetes, $48.3 \%$ were obese, and $34.6 \%$ had chronic liver disease (35). The above components of metabolic syndrome not only increase the risk of COVID-19 infection but also increase the occurrence and development of COVID-19-related complications (36), particularly those related to liver injury and the abnormal elevation of transaminase. The relationship between the components of metabolic disorder and liver injury in COVID-19 patients is discussed further below.

\section{Hypertension and liver injury in COVID-19 patients}

Hypertension is an important risk factor that affects the occurrence and progression of COVID-19 (5). At present, there is no report yet on the global incidence of hypertension in COVID-19 patients. In a cohort study of patients with COVID-19 and chronic metabolic diseases, COVID-19 had the highest incidence in patients with hypertension and 49.7\% of COVID-19 patients suffer from hypertension (36). Hypertension is associated with the abnormal activation of the renin-angiotensin system (RAS) and the decreased expression of ACE2 (37,38). Angiotensin II (AngII) is a key regulator of the RAS that can promote oxidative stress and the inflammation of vascular endothelial cells and smooth muscle cells to promote atherosclerosis (38). ACE2 is a negative feedback regulator of the RAS that protects blood vessels and organs by degrading the heptapeptide angiotensin 1-7 produced by AngII to produce antiinflammatory and vasodilating functions (37). Patients with hypertension have decreased levels of ACE2. As SARSCoV-2 invades the body through ACE2 receptors, these observations contradict the conclusion that patients with hypertension are susceptible to COVID-19. Alternatively, it has been proposed that the prognosis of COVID-19 patients may be related to decreases in ACE2 activity in patients with hypertension. SARS-CoV-2 infection reduces the activity of ACE2 activity and its receptors. This can disrupt the ACE2 pathway leading to the occurrence and progression of COVID-19 (39).

Zheng et al. suggested that hypertension may increase the risk of liver injury in COVID-19 patients (40), and ACE2 is undoubtedly involved in this process. ACE2 plays an important role in regulating the function of the cardiovascular, renal, and liver systems (37). The expression of ACE2 receptors is significantly higher in bile duct cells than in hepatocytes. There is almost no evidence of biliary injury being manifested as increases in $\gamma$-glutamyl transferase and alkaline phosphatase. Thus, liver injury in hypertensive patients with COVID-19 is not necessarily related to the direct action of SARS$\mathrm{CoV}-2$ on ACE2 receptors.

Liver injury in COVID-19 patients may be due to secondary factors, such as the activation of systemic inflammatory reactions in the case of hypertension. Yang et al. conducted a retrospective study of 251 COVID-19 patients, 126 of whom had hypertension. The study showed that COVID-19 patients with hypertension had higher levels of IL-6 and a higher sensitivity to C-reactive protein and procalcitonin than non-hypertensive patients (41). These data suggest that COVID-19 patients with hypertension have a more severe systemic inflammatory response than those without hypertension. This response can affect liver metabolism and cause secondary liver injury or multiple organ dysfunction in severe cases.

\section{Diabetes and liver injury in COVID-19 patients}

Diabetes is also a risk factor for COVID-19 (42-44). Kumar et al. showed that up to $73.3 \%$ of patients with diabetes mellitus complicated with COVID-19 have liver injury, which indicates that diabetes is one of the risk factors 
of liver injury in COVID-19 (45). In the US, another study involving 2,237 cases of COVID-19 showed that of 886 patients with diabetes, $16.7 \%$ had a more than 2 -fold increase in ALT and that diabetes was an independent-related factor for abnormally elevated ALT (46).

Currently, most reports on liver injury in patients with diabetes mellitus complicated with COVID-19 have rarely found abnormal increases in ALT and TBIL. The mechanism of diabetes on liver injury in patients with COVID-19 remains unclear. We hypothesize that hyperglycemia contributes to the replication of SARS$\mathrm{CoV}-2$, which increases the extent of viral invasion of the virus. This then acts to aggravate the systemic inflammatory response, causing multiple system organ damage or failure. Additionally, diabetes is considered an autoimmune and chronic inflammatory disease. Patients with diabetes have autoimmune system dysfunction and imbalances of CD4+T/CD8+T lymphocytes, natural killer cells and B cells $(47,48)$. These factors may be further enhanced by COVID-19, resulting in systemic inflammatory reactions, an inflammatory storm, and immune dysfunction.

The liver is also an important immunomodulatory organ in the human body that participates in the phagocytosis of exogenous microorganisms and the release of cytokines. Diabetes mellitus complicated with COVID-19 may increase the immunomodulatory load of the liver. Inflammatory reactions and the inflammatory storm caused by diabetes and COVID-19 may further affect various organs, including the liver. Finally, hypoglycemic drugs, such as acarbose, may also cause abnormal increases in transaminase (49). It has also been reported that metformin can aggravate immune-mediated liver injury (50).

\section{Dyslipidemia and liver injury in COVID-19 patients}

A study of COVID-19 patients showed that dyslipidemia was the second most frequent complication after hypertension (46.8\%), and is strongly correlated with COVID-19 (35). Dyslipidemia is an important component of metabolic syndrome that is mainly characterized by abnormal increases in triglycerides (TC) and abnormal decreases in the levels of high-density lipoprotein cholesterol (HDL-c). Research has shown that after the onset of the disease, COVID-19 patients' levels of total cholesterol (TC), Triacylglycerol (TG), high-density lipoprotein cholesterol (HDL-c) and low-density lipoprotein cholesterol (LDL-c) lowered significantly. These changes were related to disease severity and the mortality $(51,52)$. The abnormal decrease in blood lipid levels is consistent with the dyslipidemia of metabolic syndrome.

There is evidence that blood lipids and cholesterol are involved in the replication and proliferation of the SARS virus in cells (53). As the SARS virus is highly homologous to SARS-CoV-2, we speculate that the abnormal decrease of blood lipid components is caused by the synthesis and packaging of virus particles using lipids while SARS$\mathrm{CoV}-2$ invading host cells. The abnormal increase in TC in patients with metabolic syndrome may create favorable conditions for SARS-CoV-2 replication. As the liver is the main site of lipid metabolism and the virus replicates in large quantities in the liver, it is reasonable to hypothesize that the virus directly causes damage to the liver and further aggravates disorders of lipid metabolism. Patients with metabolic syndrome and dyslipidemia often have liver lipid metabolic overload that may have a synergistic effect when combined with COVID-19. This may also be one of the causes of liver injury; however, studies need to be conducted to validate this theory.

\section{Obesity and liver injury in COVID-19 patients}

Obesity is an independent risk factor for COVID-19. A cohort of 342 patients with COVID-19 showed that $52 \%$ of the patients were complicated with obesity (54). Another study showed that $18.6 \%$ of COVID-19 patients with obesity had liver injury (55). The specific mechanism by which liver injury is caused in obese COVID-19 patients is unknown, but a number of potential causes may explain this relationship. First, obese patients often also suffer from non-alcoholic fatty liver disease (NAFLD) due to the accumulation of visceral fat (30). The inflammatory response of NAFLD itself can lead to chronic liver injury. On this basis, COVID-19 and the drugs used to treat COVID-19 may further aggravate liver injury. Second, obese patients with impaired ventilation can suffer from sleep apnea syndrome, which can lead to hypoxemia, which in turn results in an anoxic environment for liver metabolism and consequent liver injury (56). Third, obesity has been shown to affect the immune system (57). Adipocytes and immune cells share several similar functions and can produce inflammatory mediators (57). Obesity can lead to an imbalance in the regulation of adipocytes and the immune system (58) that may promote the occurrence of an inflammatory storm due to COVID-19, which in turn could result in multisystem organ injury. 


\section{Conclusions}

The COVID-19 pandemic has created a heavy medical burden and poses health risks to the global population. Liver injury is a common complication of COVID-19. In this article, we reviewed current research findings on liver injury in patients with COVID-19 in the context of metabolic syndrome. Both COVID-19 and metabolic syndrome cause liver damage through direct or indirect mechanisms. Therefore, when COVID-19 is combined with metabolic syndrome, it may increase the risk of liver injury, and it cannot be ruled out that the two diseases have a superimposed effect on liver injury. However, further studies need to be conducted to determine the mechanisms underpinning these observations to minimize the effects of metabolic factors on liver injury in patients with COVID-19.

\section{Acknowledgments}

Funding: This work was supported by the Joint Medical Research Project of Chongqing Municipal Science and the Technology Bureau and Health Commission (2020FYYX077).

\section{Footnote}

Reporting Checklist: The authors have completed the Narrative Review reporting checklist. Available at https:// dx.doi.org/10.21037/apm-21-1398

Conflicts of Interest: All authors have completed the ICMJE uniform disclosure form (available at https://dx.doi. org/10.21037/apm-21-1398). The authors have no conflicts of interest to declare.

Ethical Statement: The authors are accountable for all aspects of the work, including ensuring that questions related to the accuracy or integrity of any part of the work have been appropriately investigated and resolved.

Open Access Statement: This is an Open Access article distributed in accordance with the Creative Commons Attribution-NonCommercial-NoDerivs 4.0 International License (CC BY-NC-ND 4.0), which permits the noncommercial replication and distribution of the article with the strict proviso that no changes or edits are made and the original work is properly cited (including links to both the formal publication through the relevant DOI and the license). See: https://creativecommons.org/licenses/by-nc-nd/4.0/.

\section{References}

1. Huang C, Wang Y, Li X, et al. Clinical features of patients infected with 2019 novel coronavirus in Wuhan, China. Lancet 2020;395:497-506.

2. Wang D, Hu B, Hu C, et al. Clinical Characteristics of 138 Hospitalized Patients With 2019 Novel CoronavirusInfected Pneumonia in Wuhan, China. JAMA 2020;323:1061-9.

3. Wang C, Horby PW, Hayden FG, et al. A novel coronavirus outbreak of global health concern. Lancet 2020;395:470-3.

4. Gao Y, Ye LS, Du J, et al. Management of an endoscopy center during the outbreak of COVID-19: Experience from West China Hospital. World J Gastroenterol 2020;26:5749-58.

5. Asselah T, Durantel D, Pasmant E, et al. COVID-19: Discovery, diagnostics and drug development. J Hepatol 2021;74:168-84.

6. Marhl M, Grubelnik V, Magdic M, et al. Diabetes and metabolic syndrome as risk factors for COVID-19. Diabetes Metab Syndr 2020;14:671-7.

7. Bansal R, Gubbi S, Muniyappa R. Metabolic Syndrome and COVID 19: Endocrine-Immune-Vascular Interactions Shapes Clinical Course. Endocrinology 2020;161:bqaa112.

8. Yanai H. Metabolic Syndrome and COVID-19. Cardiol Res 2020;11:360-5.

9. Sharma P, Kumar A. Metabolic dysfunction associated fatty liver disease increases risk of severe Covid-19. Diabetes Metab Syndr 2020;14:825-7.

10. Saklayen MG. The Global Epidemic of the Metabolic Syndrome. Curr Hypertens Rep 2018;20:12.

11. García-García FJ, Monistrol-Mula A, Cardellach F, et al. Nutrition, Bioenergetics, and Metabolic Syndrome. Nutrients 2020;12:2785.

12. Ranasinghe $P$, Mathangasinghe $Y$, Jayawardena $R$, et al. Prevalence and trends of metabolic syndrome among adults in the asia-pacific region: a systematic review. BMC Public Health 2017;17:101.

13. Zhang H, Liao YS, Gong J, et al. Clinical characteristics and risk factors for liver injury in COVID-19 patients in Wuhan. World J Gastroenterol 2020;26:4694-702.

14. Sun JK, Liu Y, Zou L, et al. Acute gastrointestinal injury in critically ill patients with COVID-19 in Wuhan, China. World J Gastroenterol 2020;26:6087-97. 
15. Hajifathalian K, Mahadev S, Schwartz RE, et al. SARSCOV-2 infection (coronavirus disease 2019) for the gastrointestinal consultant. World J Gastroenterol 2020;26:1546-53.

16. Beyerstedt S, Casaro EB, Rangel ÉB. COVID-19: angiotensin-converting enzyme 2 (ACE2) expression and tissue susceptibility to SARS-CoV-2 infection. Eur J Clin Microbiol Infect Dis. 2021;40:905-919.

17. Zheng YY, Ma YT, Zhang JY, et al. COVID-19 and the cardiovascular system. Nat Rev Cardiol 2020;17:259-60.

18. Madjid M, Safavi-Naeini P, Solomon SD, Vardeny O. Potential Effects of Coronaviruses on the Cardiovascular System: A Review. JAMA Cardiol 2020;5:831-40.

19. Guan WJ, Ni ZY, Hu Y, et al. China Medical Treatment Expert Group for C. Clinical Characteristics of Coronavirus Disease 2019 in China. N Engl J Med 2020;382:1708-20.

20. Schattenberg JM, Labenz C, Worns MA, et al. Patterns of liver injury in COVID-19 - a German case series. United European Gastroenterol J 2020;8:814-9.

21. Guo T, Fan Y, Chen M, et al. Cardiovascular Implications of Fatal Outcomes of Patients With Coronavirus Disease 2019 (COVID-19). JAMA Cardiol 2020;5:811-8.

22. Alqahtani SA, Schattenberg JM. Liver injury in COVID-19: The current evidence. United European Gastroenterol J 2020;8:509-19.

23. Uhlén M, Fagerberg L, Hallstrom BM, et al. Proteomics. Tissue-based map of the human proteome. Science 2015; 347: 1260419.

24. Paizis G, Tikellis C, Cooper ME, et al. Chronic liver injury in rats and humans upregulates the novel enzyme angiotensin converting enzyme 2. Gut 2005;54:1790-6.

25. Hoffmann M, Kleine-Weber H, Schroeder S, et al. SARSCoV-2 Cell Entry Depends on ACE2 and TMPRSS2 and Is Blocked by a Clinically Proven Protease Inhibitor. Cell 2020;181:271-280.e8.

26. Gonzalez FJ, Xie C, Jiang C. The role of hypoxiainducible factors in metabolic diseases. Nat Rev Endocrinol 2018;15:21-32.

27. Liu J, Li S, Liu J, et al. Longitudinal characteristics of lymphocyte responses and cytokine profiles in the peripheral blood of SARS-CoV-2 infected patients. EBioMedicine 2020;55:102763.

28. Boeckmans J, Rodrigues RM, Demuyser T, et al. COVID-19 and drug-induced liver injury: a problem of plenty or a petty point? Arch Toxicol 2020;94:1367-9.

29. Feng G, Zheng KI, Yan QQ, et al. COVID-19 and Liver Dysfunction: Current Insights and Emergent Therapeutic
Strategies. J Clin Transl Hepatol 2020;8:18-24.

30. Portincasa P, Krawczyk M, Smyk W, et al. COVID-19 and non-alcoholic fatty liver disease: Two intersecting pandemics. Eur J Clin Invest 2020;50:e13338.

31. Mendez-Sanchez N, Valencia-Rodriguez A, Qi X, et al. What Has the COVID-19 Pandemic Taught Us so Far? Addressing the Problem from a Hepatologist's Perspective. J Clin Transl Hepatol 2020;8:0024.

32. Carr DB, Utzschneider KM, Hull RL, et al. Intraabdominal fat is a major determinant of the National Cholesterol Education Program Adult Treatment Panel III criteria for the metabolic syndrome. Diabetes 2004;53:2087-94.

33. Executive Summary of The Third Report of The National Cholesterol Education Program (NCEP) Expert Panel on Detection, Evaluation, And Treatment of High Blood Cholesterol In Adults (Adult Treatment Panel III). Expert Panel on Detection, Evaluation, and Treatment of High Blood Cholesterol in Adults. JAMA. 2001;285:2486-97.

34. Costa FF, Rosario WR, Ribeiro Farias AC, et al. Metabolic syndrome and COVID-19: An update on the associated comorbidities and proposed therapies. Diabetes Metab Syndr 2020;14:809-14.

35. Garg S, Kim L, Whitaker M, et al. Hospitalization Rates and Characteristics of Patients Hospitalized with Laboratory-Confirmed Coronavirus Disease 2019 COVID-NET, 14 States, March 1-30, 2020. MMWR Morb Mortal Wkly Rep 2020;69:458-64.

36. Ghoneim S, Butt MU, Hamid O, et al. The incidence of COVID-19 in patients with metabolic syndrome and non-alcoholic steatohepatitis: A population-based study. Metabol Open 2020;8:100057.

37. Tikellis C, Bernardi S, Burns WC. Angiotensin-converting enzyme 2 is a key modulator of the renin-angiotensin system in cardiovascular and renal disease. Curr Opin Nephrol Hypertens 2011;20:62-8.

38. Chen XL, Tummala PE, Olbrych MT, et al. Angiotensin II induces monocyte chemoattractant protein-1 gene expression in rat vascular smooth muscle cells. Circ Res 1998;83:952-9.

39. Cheng H, Wang Y, Wang GQ. Organ-protective effect of angiotensin-converting enzyme 2 and its effect on the prognosis of COVID-19. J Med Virol 2020;92:726-30.

40. Zheng Z, Peng F, Xu B, et al. Risk factors of critical \& mortal COVID-19 cases: A systematic literature review and meta-analysis. J Infect 2020;81:e16-25.

41. Yang G, Tan Z, Zhou L, et al. Effects of Angiotensin II Receptor Blockers and ACE (Angiotensin-Converting 
Enzyme) Inhibitors on Virus Infection, Inflammatory

Status, and Clinical Outcomes in Patients With

COVID-19 and Hypertension: A Single-Center

Retrospective Study. Hypertension 2020;76:51-8.

42. Mantovani A, Byrne CD, Zheng MH, et al. Diabetes as a risk factor for greater COVID-19 severity and in-hospital death: A meta-analysis of observational studies. Nutr Metab Cardiovasc Dis 2020;30:1236-48.

43. Dennis JM, Mateen BA, Sonabend R, et al. Type 2 Diabetes and COVID-19-Related Mortality in the Critical Care Setting: A National Cohort Study in England, March-July 2020. Diabetes Care 2021;44:50-7.

44. Targher G, Mantovani A, Wang XB, et al. Patients with diabetes are at higher risk for severe illness from COVID-19. Diabetes Metab 2020;46:335-7.

45. Kumar A, Kumar P, Dungdung A, et al. Pattern of liver function and clinical profile in COVID-19: A crosssectional study of 91 patients. Diabetes Metab Syndr 2020;14:1951-4.

46. Phipps MM, Barraza LH, LaSota ED, et al. Acute Liver Injury in COVID-19: Prevalence and Association with Clinical Outcomes in a Large U.S. Cohort. Hepatology 2020;72:807-17.

47. Pietropaolo M, Barinas-Mitchell E, Kuller LH. The heterogeneity of diabetes: unraveling a dispute: is systemic inflammation related to islet autoimmunity? Diabetes 2007;56:1189-97.

48. Guzmán-Flores JM, Lopez-Briones S. Cells of innate and adaptive immunity in type 2 diabetes and obesity. Gac Med Mex 2012;148:381-9.

49. Chao CT, Wang J, Huang JW, et al. Acarbose Use and Liver Injury in Diabetic Patients With Severe Renal Insufficiency and Hepatic Diseases: A Propensity ScoreMatched Cohort Study. Front Pharmacol 2018;9:860.

Cite this article as: Li R, Tang Y, Liang M, Ding J. Liver injury in COVID-19 patients with metabolic syndrome-a narrative review. Ann Palliat Med 2021;10(7):8264-8270. doi: 10.21037/apm21-1398
50. Volarevic V, Misirkic M, Vucicevic L, et al. Metformin aggravates immune-mediated liver injury in mice. Arch Toxicol 2015;89:437-50.

51. Fan J, Wang H, Ye G, et al. Letter to the Editor: Lowdensity lipoprotein is a potential predictor of poor prognosis in patients with coronavirus disease 2019. Metabolism 2020;107:154243.

52. Wei X, Zeng W, Su J, et al. Hypolipidemia is associated with the severity of COVID-19. J Clin Lipidol 2020;14:297-304.

53. Lu Y, Liu DX, Tam JP. Lipid rafts are involved in SARSCoV entry into Vero E6 cells. Biochem Biophys Res Commun 2008;369:344-9.

54. Chen VL, Hawa F, Berinstein JA, et al. Hepatic Steatosis Is Associated with Increased Disease Severity and Liver Injury in Coronavirus Disease-19. Dig Dis Sci 2020;27:1-7.

55. Lenti MV, Borrelli de Andreis F, Pellegrino I, et al. Impact of COVID-19 on liver function: results from an internal medicine unit in Northern Italy. Intern Emerg Med 2020;15:1399-407.

56. Moser JS, Galindo-Fraga A, Ortiz-Hernandez AA, et al. Underweight, overweight, and obesity as independent risk factors for hospitalization in adults and children from influenza and other respiratory viruses. Influenza Other Respir Viruses 2019;13:3-9.

57. Martí A, Marcos A, Martinez JA. Obesity and immune function relationships. Obes Rev 2001;2:131-40.

58. Nave H, Beutel G, Kielstein JT. Obesity-related immunodeficiency in patients with pandemic influenza H1N1. Lancet Infect Dis 2011;11:14-5.

(English Language Editor: L. Huleatt) 